\title{
Planificações Parametrizadas e Kirigami: Aproximações possiveis para o Estudo é a Representação de Superfícies Curvas
}

Parameterized flattening and Kirigami: Possible Approximations to the

Study and the Representation of Curved Surfaces

\author{
> Janice de Freitas Pires \\ Universidade Federal de Pelotas, Brasil \\ janicefpires@hotmail.com
}

> Adriane Borda Almeida da Silva

Universidade Federal de Pelotas, Brasil

adribord@hotmail.com
> Gabriela Gonzales Peronti

Universidade Federal de Pelotas, Brasil

Email Address

> Mariana Reami Osamaré

Universidade Federal de Pelotas, Brasil

Email Address

\begin{abstract}
This paper demonstrates the process of generation of physical paper models from digital models of curved surfaces through the digital technique for approximately flattening. A collection of these models, based on works of architecture, is being produced as a didactic activity within a discipline of graphic and digital geometry in the curriculum of architecture. The study allowed the identification of parameters to improve the process of generating such models and recognize the kirigami technique as an alternative for the representation of non-developable rectilinearsurfaces, previously not included for the generation of physical models in the didactic activities in question.
\end{abstract}

Keywords: Parameterized flattening; curved surfaces; paper models; kirigami. Planificaçôes parametrizadas; superfícies curvas; modelos em papel.

\section{Introdução}

Em processos tradicionais de aprendizagem no âmbito de disciplinas de Geometria Descritiva, as técnicas de planificação aproximada de superfícies curvas possibilitam a execução de modelos físicos. No contexto trabalhado, por muito tempo, no âmbito de práticas de ensino, foram utilizados processos manuais para a geração de maquetes em papel através de tais técnicas. Entretanto, considerandose o trabalho exaustivo para poder representar formas complexas, os exercícios limitavam-se à experiência com um tipo limitado de superfícies, tais como as cilíndricas, as cônicas e demais superfícies clássicas de revolução, nos termos de Rodrigues (1960).

Atualmente, valendo-se de técnicas automatizadas e digitais de planificação, tem sido possível abordar superfícies complexas no âmbito das mesmas práticas didáticas. Neste contexto formativo, tem-se sempre o propósito de utilizar-se de obras de arquitetura de valor reconhecido. Os estudantes têm realizado experiências de representaçáo, as quais buscam contemplar, ao mesmo tempo, a aquisiçâao de habilidades conceituais e procedimentais no âmbito da geometria, a apropriação de tecnologias digitais e o reconhecimento de repertórios arquitetônicos para o projeto.

As técnicas de planificação digitais tem permitido agilizar as representaçôes de superfícies circulares de revolução, e avançar para outros tipos de superfícies, motivando os estudantes para a obtençâo dos modelos físicos a partir dos digitais. No entanto, tem-se observado a necessidade de atentar para o processo de delimitação dos parâmetros adotados para as planificaçôes. Tais parâmetros referem-se, por exemplo, ao controle do número e da forma de polígonos atribuídos à superfície, os quais determinam resultados mais ou menos aproximados à forma curva a ser representada.

A experiência didática também exigiu atentar especialmente para o caso das superfícies retilíneas não planificáveis, geradas por retas reversas, caso em que o controle do número e da forma de polígonos é ainda mais determinante da viabilidade de executar manualmente um modelo físico em papel.

Em busca de referenciais para o aperfeiçoamento destas práticas, identificou-se na técnica do Kirigami tridimensional, a possibilidade de apoio. Razani (1993) demonstra o uso desta técnica aplicada às superfícies em questáo. $\mathrm{O}$ Kirigami associa o procedimento de dobra, próprio da técnica milenar do Origami, ao procedimento de corte para a montagem da forma tridimensional (Ueno e Nascimento, 2009). Assim como as maquetes em papel, um Kirigami tridimensional também se utiliza de técnicas de planificaçáo para sua composição. Além disto, faz-se necessário identificar as regras de organização destes elementos no plano e de configuração de suas dobras para determinar a tridimensionalidade, específicas para cada tipo de superfície a ser representada. Considerou-se, no âmbito deste trabalho, que a compreensão da lógica de estruturação de Kirigamis tridimensionais auxilia o estudo para o aprimoramento do processo de geraçáo de modelos físicos em papel para representar as superfícies retilíneas não planificáveis.

Por se tratar de estágios iniciais de formação, o objetivo das representaçōes é de estudos volumétricos para o "croqui", físico e tridimensional, considerando-se que tanto o processo de planificaçáo aproximada quanto o Kirigami tem o propósito de 

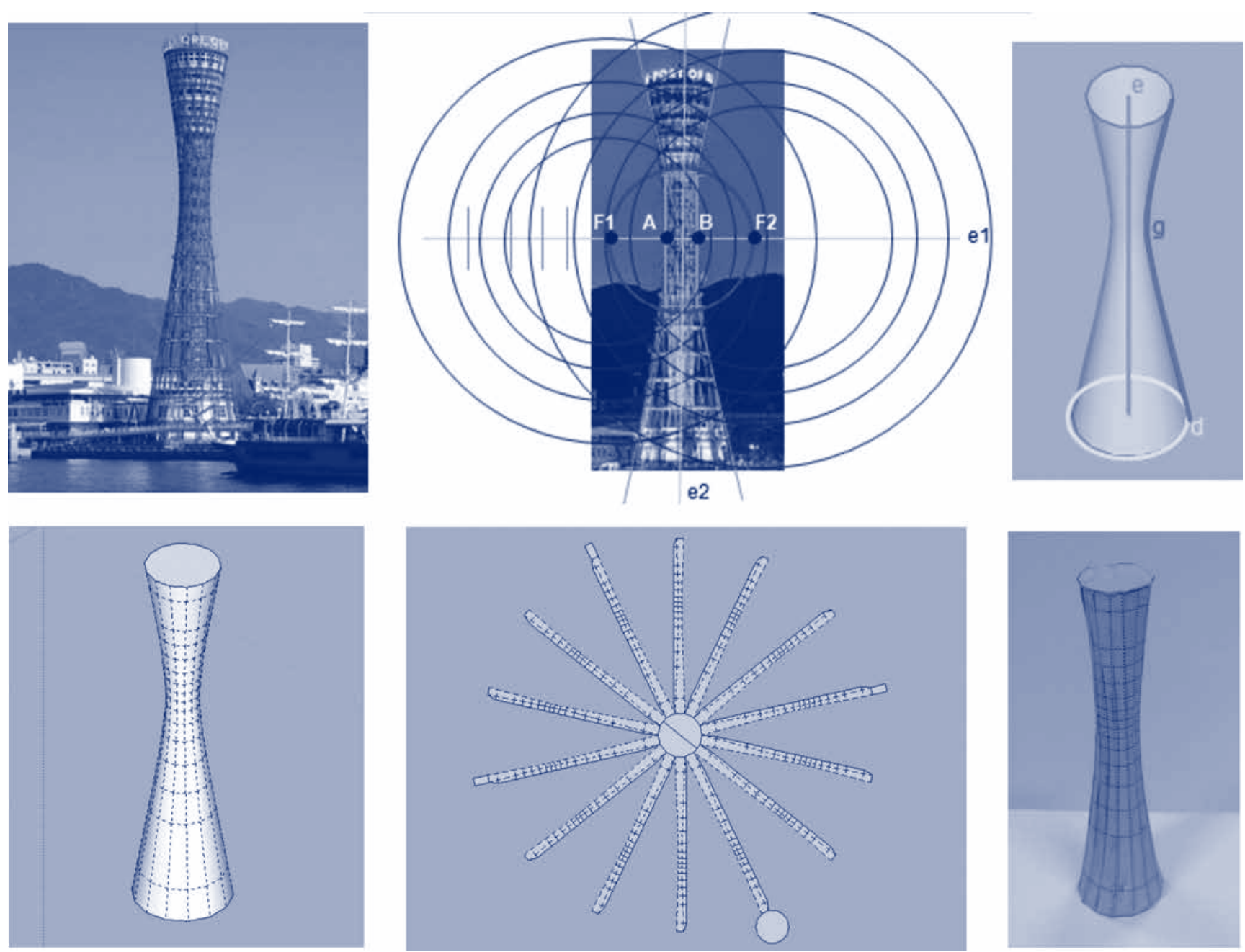

Figura 1: Etapas propostas para o estudo de superfícies curvas. Fonte: https:// www.flickr.com/photos/raktargy/6998949822/sizes/l/; modelos produzidos pela estudante Graziele Leitzke, na disciplina Geometria Gráfica e Digital 3, da FAURB/ UFPel, $1^{\circ}$ semestre de 2014, representativos da obra Torre de Kobe, no Japão.

auxiliar na percepção da forma. Busca-se assim observar se estas atividades irão permitir aos estudantes ampliar o repertório de formas a representar, já que o processo de planificaçáo tradicional apresentado em Rodrigues (1960) é aplicado para apenas a classe de superfícies curvas circulares de revolução.

\section{Metodologia}

O estudo vem sendo desenvolvido para apoiar as atividades didáticas da disciplina de Geometria Gráfica e Digital 3 do curso de Arquitetura e Urbanismo da Universidade Federal de Pelotas, RS, Brasil. Esta disciplina abarca conceitos e procedimentos relativos às superfícies curvas, a partir de técnicas tradicionais e digitais de representaçáo de obras de arquitetura que envolvem o uso de tais superfícies, conforme descrito em Pires et al (2011; 2013). Um dos conteúdos tratado se refere à planificação aproximada dos modelos digitais de tais obras de arquitetura, com o objetivo então de construir modelos em papel. A Figura 1 ilustra cada uma das etapas de trabalho desenvolvidas pelos estudantes. Na primeira linha, da esquerda para a direita, tem-se exemplificado o resultado da etapa de seleçáo de uma obra de arquitetura a ser representada; ao lado, a exemplificação da análise geométrica da obra, na busca da identificação dos elementos principais para a sua representação; e, logo, a representação gráfica digital. Na segunda linha, também da esquerda para a direita, tem-se o resultado da etapa de determinação dos parâmetros para a planificação; ao lado, tem-se a planificação obtida automaticamente; e, logo, o resultado da etapa de construçáo do modelo físico em papel da superfície representada.

Durante o desenvolvimento das práticas propostas, em 2013 e 2014, foram sendo investigados parâmetros de adequação da modelagem geométrica das superfícies visando facilitar, qualificar e em alguns casos tornar viável a montagem dos modelos em papel. Tais práticas possibilitaram também desenvolver o mesmo processo para algumas superfícies da classe de superfícies quádricas em geral geradas por curvas cônicas, sob a terminologia de Rodrigues (1960). A partir do segundo semestre de 2013 passou-se a estudar então a técnica de kirigami, através do trabalho de Razani (1993). Com esta técnica avançou-se na execução dos modelos em papel, abarcando a representação da classe de superfícies retilíneas não planificáveis, como paraboloides hiperbólicos, cilindroides, conoides e helicoides. 
O estudo desenvolve-se através das seguintes etapas:

a. Reconhecimento do processo de planificação aproximada de superfícies curvas circulares de revolução, por meios tradicionais, apoiando-se em Rodrigues (1960), e da técnica de Kirigami para a confecção manual de superfícies retilíneas não planificáveis, apoiando-se em Razani (1993); Nesta etapa estudou-se a classificação dada por Rodrigues (1960) para as superfícies curvas, particularizando-se a classificação da classe de superfícies Propriamente Curvas Circulares de Revolução (Esfera, Elipsoides alongado e achatado, Paraboloide e Hiperboloide de Revolução) e Quádricas em Geral de primeira espécie (Elipsoides, Paraboloides e Hiperboloides Elípticos). Esta classificação adotada pelo autor restringe-se às superfícies geradas a partir de curvas cônicas. O reconhecimento da técnica do Kirigami está sendo feita através de análises geométricas sobre as representaçôes planas apresentadas por Razani (1993), para as superfícies de paraboloides hiperbólicos e cilindroides. Nas análises, particularizam-se os entes geométricos e os conceitos de composição geométrica, tais como das proporçóes entre todas as partes da representação. Analisam-se as relaçóes destas informaçôes com as do modelo tridimensional gerado.

b. Apropriação por meios digitais do processo de planificação aproximada de superfícies curvas, com o uso de plug-ins de planificação e para a técnica do Kirigami, envolvendo a planificação e a configuraçáo de dobras e cortes entre os elementos da superfície. Nesta etapa, manteve-se o uso da ferramenta de modelagem tridimensional SketchUp adotada como meio de representação nas práticas da disciplina. Os exercícios de planificação aproximada das superfícies curvas de revolução, inicialmente, eram desenvolvidos exclusivamente por técnicas tradicionais. Manualmente, a geometria da planificaçáo é determinada a partir de linhas poligonais as quais formam superfícies planas inseridas em fusos. Logo, se passou a utilizar recursos digitais que automatizam o processo de planificaçáo, como o plugin Unfoldtool, disponibilizado em http://sketchuptips.blogspot. com.br/2007/08/plugin-unfoldrb.html. Tais planificaçóes, obtidas entâo a partir do meio digital, foram impressas em papel sulfite $180 \mathrm{gr}$, em formato A3, realizando-se a montagem dos modelos. Todo este processo envolveu o reconhecimento de parâmetros específicos de modelagem geométrica e de configuração para a impressão, de acordo com a escala do modelo físico a ser gerado.

c. Seleçáo de obras de arquitetura com superfícies curvas a serem representadas digitalmente;

d. Estruturação de processos de planificação aproximada de obras de arquitetura configuradas por superfícies circulares de revolução, apoiados por técnicas digitais, observando-se o controle dos parâmetros de configuração do número de polígonos das curvas diretriz e geratriz;

e. Estruturação de processos digitais de representação dos Kirigamis de obras de arquitetura configuradas por superfícies retilíneas não desenvolvíveis; f. Realização de oficinas para: a representação gráfica digital das obras selecionadas; a planificação aproximada e a representação dos Kirigamis, por técnicas digitais, das obras modeladas; e o uso de técnicas de impressão e montagem dos protótipos em papel.

\section{Resultados}

Os resultados até o momento são parciais, referindo-se principalmente aos experimentos realizados para a representação de superfícies circulares de revolução e quádricas em geral por técnicas de planificação aproximada. Tais experimentos tem propiciado controlar os parâmetros de geraçáo da forma, adequando-os para que haja uma maior aproximação da planificação à forma curva da superfície tratada, além de adequaçôes necessárias aos processos de impressão e principalmente de montagem dos modelos físicos em papel.

Os parâmetros de modelagem geométrica das superfícies propriamente curvas, estudados com tais objetivos, se referem as suas curvas diretriz (d) e geratriz (g). A Figura 2 exemplifica o exercício dos estudantes de identificação de tais curvas, para cada uma das obras selecionadas. Naquele momento, o exercício envolvia superfícies circulares de revolução (diretrizes circulares) e quádricas de primeira espécie (diretrizes elípticas). As geratrizes destas superfícies são as curvas cônicas (circunferência para a esfera, elipse para os elipsoides, parábola para os paraboloides e hipérbole para os hiperboloides).

Figura 2: Atividades desenvolvidas pelos estudantes da disciplina de Geometria Gráfica e Digital 3, FAURB/UFPel, no ano de 2013. Figura criada por autores, 2014.

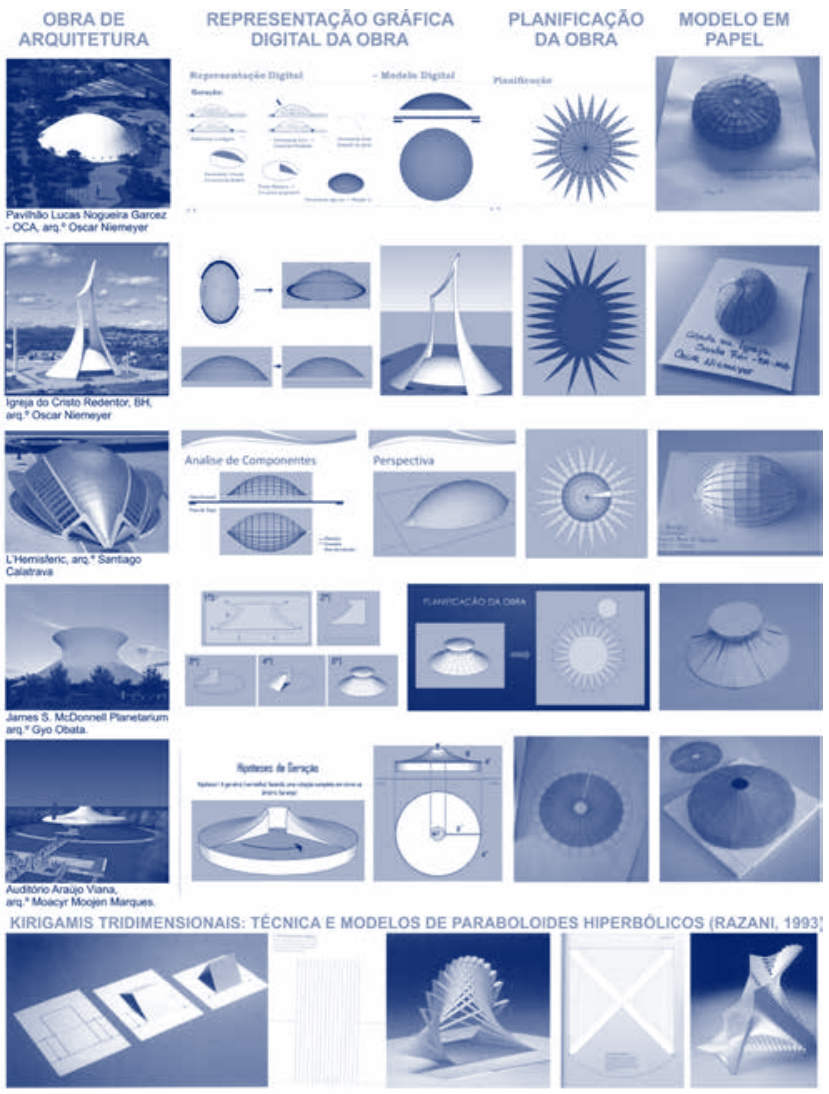


Durante a etapa de oferecimento de oficinas, desenvolvida nos anos de 2013 e 2014, foi possível identificar e adequar-se os referidos parâmetros de modelagem geométrica. A Figura 2 também inclui exemplos dos modelos físicos obtidos pelos estudantes nos dois semestres de 2013. A partir da análise destes modelos foram identificados problemas quanto: ao número inadequado de segmentos utilizados para as curvas diretriz e geratriz da superficie (ilustrado pelos modelos da primeira, terceira e quarta linha da figura 2); ocorrência de deformação na curvatura da superfície (ilustrados pelos modelos da primeira e segunda linha da figura 2); e dificuldade de uniāo entre os fusos planificados (ilustrado pelo modelo da quarta linha da figura 2). Entretanto, o modelo da última linha desta mesma figura exemplifica uma solução criada por uma estudante, a qual adiciona abas nos fusos do modelo, amenizando o problema de imprecisão da união entre eles.

A caracterização destas questóes junto aos resultados das atividades do ano de 2013 promoveu a reestruturação da prática de planificação aproximada. A Figura 3 busca comparar os exercícios desenvolvidos antes e após a proposta de reformulação das atividades. As três primeiras linhas desta Figura ilustra exemplos de trabalhos de estudantes nos quais foram identificados os problemas referentes ao: controle do número de segmentos das geratrizes e diretrizes; execução da união entre as superfícies; e a configuração da curvatura da superfície. Nas três últimas linhas desta mesma figura são apresentados os resultados após a proposta de reformulação das atividades. Foram readequados os parâmetros de modelagem e propostos recursos adicionais de apoio ao processo de montagem dos modelos, conforme descrito a seguir:

- Readequaçáo dos parâmetros de modelagem das curvas geratrizes e diretrizes:

Como parâmetros de representação das superfícies tem-se o número de segmentos das curvas geratriz e diretriz, o qual influencia diretamente em sua geometria alterando tanto a suavidade da curva e da superfície como o número de fusos que a constitui. Este número pode ser controlado em função do tipo e finalidade do modelo. Em se tratando do modelo digital, quanto maior o número de segmentos atribuído, maior será a suavidade da curva e por consequência da superfície resultante das transformaçóes aplicadas às curvas geratriz e diretriz. No entanto, quanto maior o número de segmentos da diretriz da superfície, maior será o de fusos constituintes. Isto determina as dimensóes resultantes para cada fuso a ser planificado, as quais decrescerão com o aumento do número de fusos. Isto dificulta o processo de corte dos fusos e principalmente de montagem do modelo. As curvas diretrizes são do tipo circular, para as superfícies circulares de revolução, ou elípticas, para as superfícies quádricas de primeira espécie.

É necessário então se adequar o objetivo de facilitar a montagem do modelo, com um número reduzido de fusos, ao de representar uma superfície curva de maneira aproximada sem comprometer a similaridade com a superfície.

$\mathrm{Na}$ primeira linha da Figura 3, os três modelos apresentados (obras Museu Nacional Honestino Guimarães do Arq. Oscar Niemeyer, Reichstag Dome do Arq. Norman Foster e Barcelona El Prat Airport do Arq. Ricardo Bofill Levi, respectivamente) não possuem controle adequado do número de segmentos da curva geratriz da superfície. Nesta linha, o primeiro modelo, à esquerda, foi gerado com um número baixo de segmentos de sua geratriz, o que resultou em um modelo físico pouco similar à forma a ser representada. Os dois modelos seguintes foram representados com número de segmentos alto, o que dificultou a sua montagem. Nos modelos físicos ilustrados na quarta linha da mesma figura (Water Tower, em Nizhny Novgorod, Rússia, do Arq. Vladimir Shukhov, Tornado Tower, do Arqs. Cico - Qatar e SIAT - Munique e Torre Kobe, Japão, respectivamente), o número de segmentos das geratrizes é suficiente para representá-las com suavidade e adequadamente reduzido para facilitar o processo de montagem. Nestes mesmos modelos pode-se ainda observar o controle adequado do número de segmentos da diretriz e dos fusos frente à escala do modelo físico e ao propósito de sua montagem, considerando-se o tamanho de cada fuso gerado pelo processo de planificaçáo.

A tabela 1 registra os parâmetros de controle de modelagem, identificados pela prática das atividades anteriores de planificação aproximada, considerados adequados para cada tipo de superfície da classe das propriamente curvas. Além de tais parâmetros, foram identificados os tipos de curvas disponíveis para a modelagem de cada superfície e a possibilidade de controle do número de segmentos das curvas geratrizes e diretrizes do modelo digital. Cabe destacar que dentre as curvas geratrizes das superfícies circulares de revoluçáo clássicas (esfera, elipsoides, paraboloide e hiperboloides), o programa de modelagem tridimensional Sketchup disponibiliza apenas a circunferência como primitiva de modelagem. No entanto, existe um plug-in de nome Curve Maker', que disponibiliza mais duas destas curvas: a parábola e a elipse. Estas três curvas podem ser controladas precisamente quanto ao parâmetro de número de segmentos que configura a curva. No entanto, para representar a curva hipérbole, tem sido necessário adotar um modelo de curva paramétrica, através da ferramenta de curvas Catmull Spline, disponível no mesmo plug-in.

- Identificação de estratégias para o aperfeiçoamento dos modelos físicos montados a partir das planificaçōes digitais aproximadas:

Identificou-se que em alguns modelos, mesmo tendo sido controlado adequadamente o número de segmentos da geratriz, sua curvatura se deformou durante o processo de montagem, conforme ilustrado pelos modelos da terceira linha da Figura 3. Considerando-se a dificuldade de obter, com o modelo físico, a curvatura exata da geratriz das superfícies, buscou-se adicionar uma estrutura interna ao modelo, através de no mínimo dois gabaritos planos com o formato desta curva, dispostos ortogonalmente. Os resultados após esta proposta estão ilustrados pelos modelos da última linha da Figura 3.

Incorporou-se a prática apresentada pela estudante que imprimiu duas planificaçóes da superfície e utilizou a segunda delas para unir internamente os fusos planificados, modelo exemplificado à direita da penúltima linha da Figura 3. No entanto, optou-se por representar abas de uniáo entre os fusos, as quais possuem a mesma função apresentada na proposta da estudante. As dificuldades anteriormente encontradas pelos estudantes podem ser observadas através dos exercícios exemplificados na segunda linha da Figura 3. Os resultados após a reformulação estão ilustrados na penúltima linha desta mesma figura.

1 http://www.drawmetal.com/curvemaker. 
Tabela 1: Parâmetros de modelagem geométrica das superfícies curvas de revolução, identificados para gerar modelos em papel.

\begin{tabular}{|c|c|c|c|c|c|c|c|}
\hline \multicolumn{8}{|c|}{$\begin{array}{c}\text { Parâmetros de modelagem indicados para Planificação Aproximada } \\
\text { da Superficie e Construção de Modelo em Papel Planificações } \\
\text { inseridas em papel Aᄏ }\end{array}$} \\
\hline \multirow[t]{2}{*}{ Superfícies } & \multirow{2}{*}{$\begin{array}{c}\text { Número de } \\
\text { Segmentos } \\
\text { da Diretriz }\end{array}$} & \multicolumn{2}{|c|}{$\begin{array}{l}\text { Número de } \\
\text { Segmentos } \\
\text { da Geratriz }\end{array}$} & \multicolumn{2}{|c|}{$\begin{array}{l}\text { Tipo de Curva de } \\
\text { modelagem }\end{array}$} & \multicolumn{2}{|c|}{$\begin{array}{l}\text { Possibilidade } \\
\text { de controle } \\
\text { preciso do } \\
\text { número de } \\
\text { segmentos }\end{array}$} \\
\hline & & 10 & 20 & $\begin{array}{c}\text { Curva } \\
\text { livre ou } \\
\text { paramé- } \\
\text { trica } \\
\end{array}$ & $\begin{array}{c}\text { Circun- } \\
\text { fe-rência, } \\
\text { Elipse, } \\
\text { Parábola }\end{array}$ & SIM & NÃO \\
\hline $\begin{array}{c}\text { Elipsoides } \\
\text { alongado, } \\
\text { achatado e } \\
\text { elíptico }\end{array}$ & $\mathrm{X}$ & & $\mathrm{x}$ & & $x$ & $\mathrm{x}$ & \\
\hline $\begin{array}{l}\text { Paraboloides } \\
\text { circular e } \\
\text { elíptico }\end{array}$ & $\mathrm{X}$ & $\mathrm{X}$ & & & $\mathrm{x}$ & $\mathrm{x}$ & \\
\hline $\begin{array}{l}\text { Hiperboloides } \\
\text { circular e } \\
\text { elíptico }\end{array}$ & $\mathrm{X}$ & $\mathrm{X}$ & & $\mathrm{X}$ & & $\mathrm{x}$ & \\
\hline Esfera & $\mathrm{X}$ & & $\mathrm{x}$ & & $\mathrm{x}$ & $\mathrm{x}$ & \\
\hline
\end{tabular}

Fonte: Tabela elaborada pelos autores, 2014.

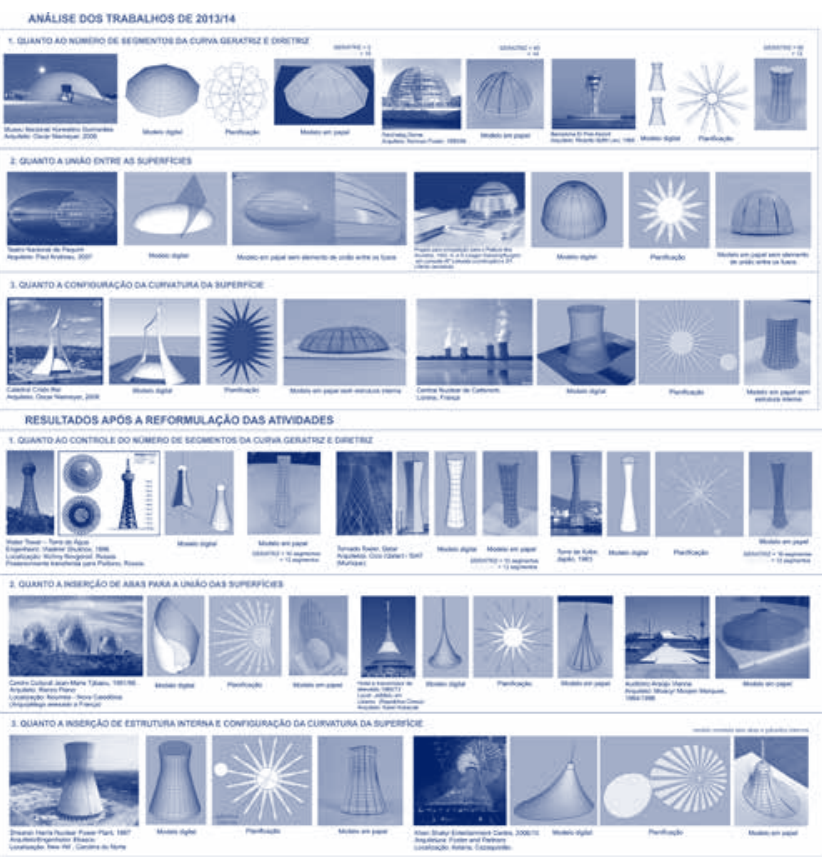

Figura 3: Modelos digitais e físicos desenvolvidas pelos estudantes da disciplina de Geometria Gráfica e Digital 3, FAURB/UFPel, nos anos de 2013 e 2014. Figura criada por autores, 2014.

Os resultados referentes a modelagem física das superfícies retilíneas não planificáveis através da técnica de kirigami ainda são iniciais. Até o momento foi analisado um tipo de kirigami proposto por Razani, ilustrado na primeira linha da figura 4. Esta análise ainda se encontra em desenvolvimento, visto que se identificou a dificuldade em analisar o kirigami referido pelo fato de ser constituído por dois hipotéticos parabolóides que se interceptam e são secionados. $\mathrm{O}$ propósito de obter uma regra geométrica que associasse a projeção do paraboloide com sua planificação e suas dimensôes no espaço tridimensional não foi ainda alcançado. A partir dos estudos do referido kirigami e de um modelo digital de uma superfície de paraboloide hiiperbólico de uma obra de arquitetura, desenvolvido em 2012 na mesma disciplina, foi gerado um kirigami por técnicas digitais e por modelagem física, conforme ilustrado nas segunda e terceira linha da figura 4. Dessa maneira, constitui-se uma metodologia para criar tais kirigamis a partir de modelos digitais de paraboloides hiperbólicos. Esta metodologia irá ser inserida na disciplina de Geometria Gráfica e Digital 3 a partir deste segundo semestre de 2014.

Segue-se com o estudo da técnica do Kirigami, buscando-se compreender as regras geométricas envolvidas na transformação dos elementos contidos no plano para a execução da volumetria das formas. Tem-se o propósito de avançar na sistematização de uma metodologia que permita representar uma classe mais abrangente de superfícies curvas e obras de arquitetura associadas e elas.

A metodologia adotada para a geração dos modelos em papel das superfícies circulares de revoluçáo tem promovido: o interesse dos estudantes para a representação das formas curvas; a compreensão dos elementos integrantes destas formas e de seus processos de geração; e a ampliaçáo do raciocínio espacial dos estudantes, habilitando-os para uma maior liberdade formal.

Considera-se que este estudo possa construir referenciais teóricos e metodológicos para apoiar, futuramente, o investimento na estruturação de técnicas parametrizadas de planificaçóes e de Kirigamis tridimensionais, as quais estimulariam a experimentação sobre a forma a partir da variação de modelos paramétricos.

\section{Conclusão}

A metodologia traçada e as atividades desenvolvidas possibilitaram: avaliar e reestruturar práticas didáticas a serem inseridas em disciplinas de geometria em estágios iniciais da formação em arquitetura; avançar em atividades de extensão, propiciando através das oficinas, a construção de conhecimento em geometria e tecnologia; de pesquisa, ao promovem a análise, revisão e readequaçóes das metodologias propostas que efetivem a inserção da tecnologia digital no projeto de arquitetura.

\section{Acknowledgments}

Agradecemos aos estudantes que cursaram a disciplina Geometria Gráfica e Digital 3, da Faculdade de Arquitetura e Urbanismo da Universidade Federal de Pelotas, em 2013 e 2014, pela contribuição dada a este trabalho.

\section{References}

Pires, J. F.; Nunes, C. S.; Vasconselos, T.; Silva, A. B. A. (2011). Trajetórias de Geometria na Arquitetura. In: XX GRAPHICA Simpósio Nacional de Geometria Descritiva e Desenho Técnico e International Conference on Graphics Engineering for Arts and Design, 2011, Rio de Janeiro. Anais...Rio de Janeiro: UFRJ, Escola de Belas Artes. 01-12. 

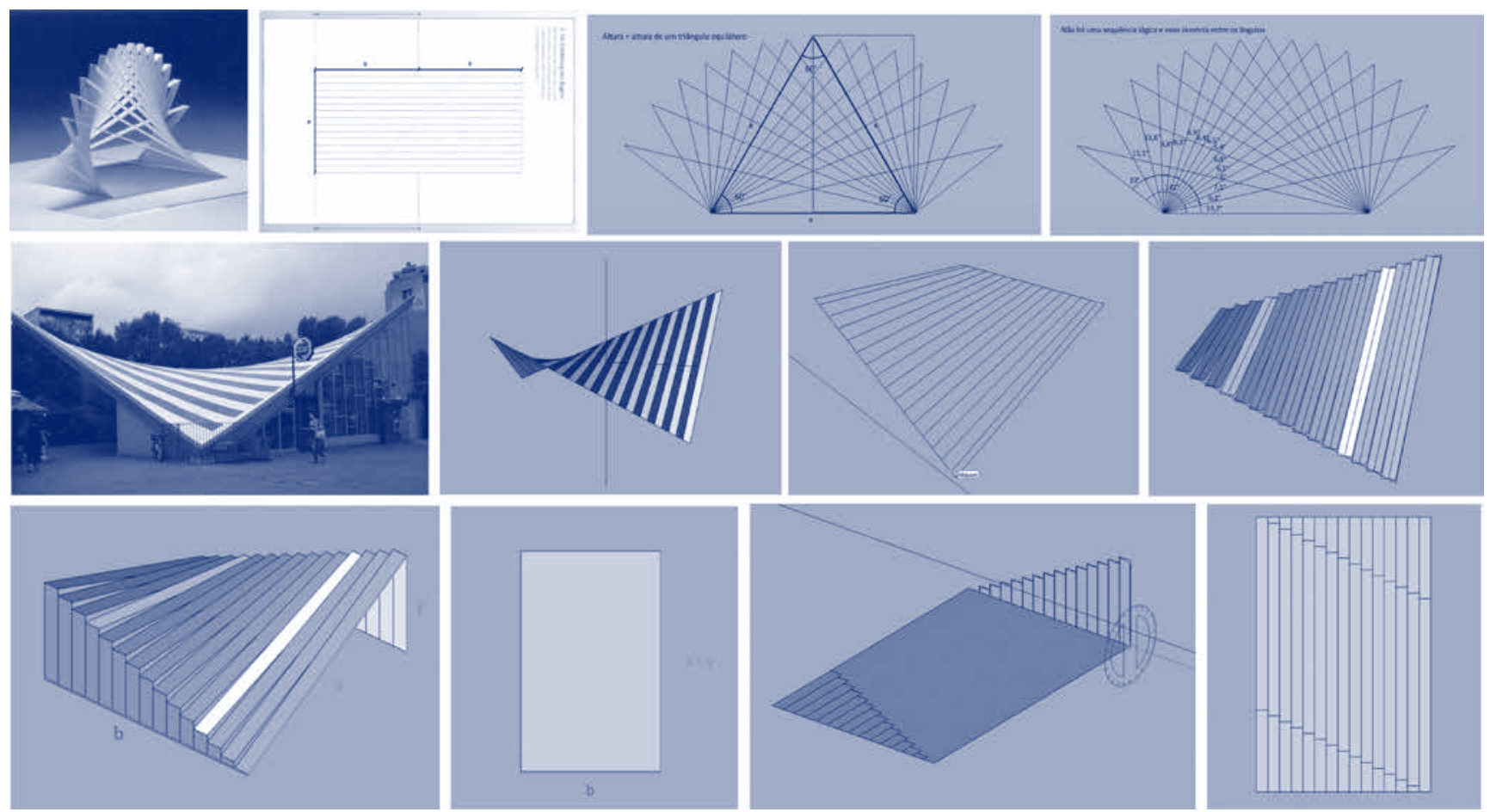

Razani, R. Phantastische Papierarbeiten. Faltschnittkarten selbermachen. Mit Anleitungen und Plänen in Originalgröße. Augustus Verlag: Augsburg, 1993. $81 \mathrm{p}$.

Rodrigues, Á. Geometria Descritiva: Projetividades, Curvas e Superficies. 1a ed. Rio de Janeiro: Ao Livro Técnico Ltda, 1960.

Figura 4: Análise geométrica do kirigami desenvolvido por Razani (1993) e proposta de modelagem geométrica e planificação do kirigami de uma obra de arquitetura (Estação de Trem Warzsawa Ochota (1963) na Polônia, modelo digital desenvolvido pela estudante Aline Moraes, em 2012). Imagem criada pelos autores. 